This item was submitted to Loughborough's Research Repository by the author.

Items in Figshare are protected by copyright, with all rights reserved, unless otherwise indicated.

\title{
The development of a virtual cycling simulator
}

PLEASE CITE THE PUBLISHED VERSION

http://dx.doi.org/10.1007/978-3-540-73011-8_18

PUBLISHER

(C) Springer

VERSION

AM (Accepted Manuscript)

PUBLISHER STATEMENT

This work is made available according to the conditions of the Creative Commons Attribution-NonCommercialNoDerivatives 4.0 International (CC BY-NC-ND 4.0) licence. Full details of this licence are available at: https://creativecommons.org/licenses/by-nc-nd/4.0/

\section{LICENCE}

CC BY-NC-ND 4.0

\section{REPOSITORY RECORD}

Tang, Yuk-Ming, Mathew Ho-Cheong Tsoi, Daniel Tik-Pui Fong, Po-Yee Pauline Lui, Kin-Chuen Hui, and KaiMing Chan. 2019. "The Development of a Virtual Cycling Simulator". figshare.

https://hdl.handle.net/2134/21256. 


\title{
The Development of a Virtual Cycling Simulator
}

\author{
Yuk-Ming Tang ${ }^{1}$, Mathew Ho-Cheong Tsoi ${ }^{1}$, Daniel Tik-Pui Fong ${ }^{1}$, \\ Pauline Po-Yee Lui ${ }^{1}$, Kin-Chuen Hui ${ }^{2}$, and Kai-Ming Chan ${ }^{1}$ \\ ${ }^{1}$ Department of Orthopaedics and Traumatology, \\ The Chinese University of Hong Kong, \\ Shatin, Hong Kong \\ \{ymtang, mathew, dfong, pauline, kmchan\}@ort.cuhk.edu.hk \\ ${ }^{2}$ Department of Mechanical and Automation Engineering, \\ The Chinese University of Hong Kong, \\ Shatin, Hong Kong \\ \{kchui\}@mae.cuhk.edu.hk
}

\begin{abstract}
Cycling is one of the current thirteen elite sports in Hong Kong. Despite cycling is one of the well known activities in the world and has numerous advantages for health, it is still far from popular in Hong Kong. In this research, a virtual cycling simulator is developed for exercise and entertainment purpose, and for promoting the cycling activity. The hardware of the cycling simulator consists of four major units including a bike platform, an actuation unit, a sensing unit and a display unit. The control system receives signals from the sensing unit and controls the motions of the actuation unit. It also computes and renders the virtual environment in real-time thereby providing the experience of cycling on different terrain models.
\end{abstract}

Keywords: Virtual cycling, virtual reality, entertainment.

\section{Introduction}

In 2006, Hong Kong cycling team has achieved their best results in international cycling competition. Two gold prizes were won in the $15^{\text {th }}$ Asian Games DOHA 2006. The significance of cycling as one of the vital exercise in Hong Kong was awakened once again. Cycling includes track cycling, road cycling and mountain bike. It has numerous advantages in health. This includes increasing fitness, lowering risk of heart attack, shedding weight and reducing stress [1].

Despite cycling is one of the well known sport activities in the world and is a good exercise for maintaining body health, it is still far from popular. It is because a large area is required for cycling. Due to the popularity of motor vehicles, recent figures [2] indicate that there is a decline in the distance traveled by cycling. Hong Kong as a small city with very high population density, the area which is suitable for cycling is very limited. Another concern of cycling is the safety problem. It is a high speed activity which is relatively dangerous comparing with the other outdoor activities 
such as hiking, camping and fishing. To ensure safety during cycling, good preparation and equipments are required.

Over the past two decades, the techniques for developing virtual reality (VR) environment have been widely used in online games. A popular approach for generating VR effect is to use the Virtual Reality Modeling Language (VRML) [3]. Although games based on VRML [4-5] provide a platform for entertainment, the online games developed using VRML are controlled using standard devices (e.g. mouse). It is still far from reality. In addition, the online games have no significant effect in promoting exercises or providing any physical exercise effect. Recently, racer mate [6] has developed a multi-rider system to provide a new method of indoor group bike training and racing. Race mate [7] has also developed an indoor virtual cycling device that is integrated with a simulation program and a graphical display. The indoor virtual cycling environment provides a solution to solve the space limitation problem as well as the safety problem. Carraro [8] has developed a bicycling simulator called Peloton. Peloton enables sharing of the virtual space and exercise with other partners. The simulators are connected through the World Wide Web. VRML is adopted for creating the virtual environment. The virtual environment is generated by synthesizing the environment created using VRML and videos of the real environment [9]. Kwon et al. [10] developed an interactive bicycle simulator. The rider can have the experience of riding in the campus of the Korea Advanced Institute of Science and Technology (KAIST). Nevertheless, three computers are required to control the system.

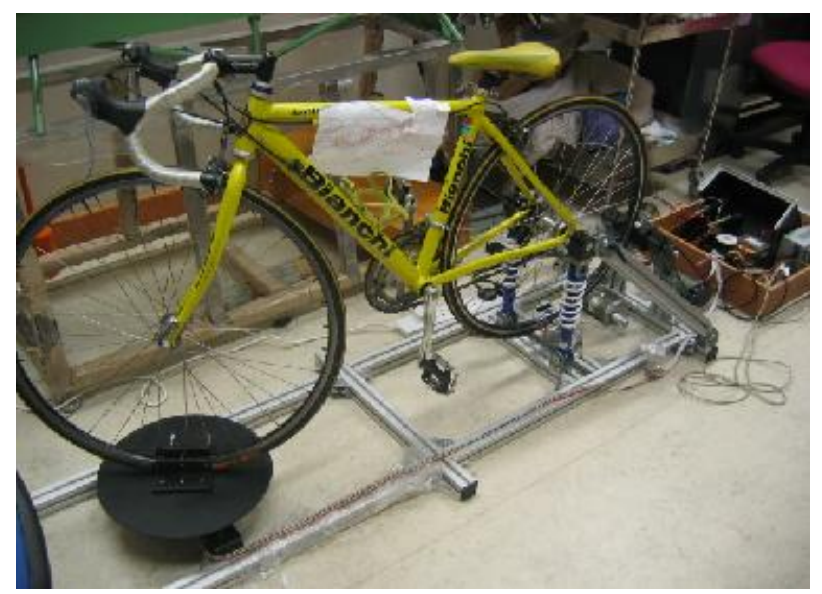

Fig. 1. The setup of the virtual cycling simulator.

In this research, a virtual cycling simulator incorporating dynamic changes and interactions between the virtual environment and the bike has been developed. The dynamic changes of the virtual environment include the frictional force between the ground and the bike, speeds of the bike riding up/ down hill. In addition, the simulator provides a stereoscopic display of the virtual environment to give an immersive cycling experience. Our work is similar to that of the CompuTrainer [7] and [10]. The objective is to provide a platform for entertainment and for promoting cycling 
exercise. With the effect of immersion and coaching of the virtual environment, it is believed that the motivation of the user to go real cycling can be enhanced [11] as well. In our system, only one PC is required. Figure 1 shows the setup of the simulator.

This paper is organized as follows. The architecture and features of the virtual cycling simulator are described in Section 2. In Section 3 the hardware setup of the simulator is described. In Section 4 we describe the software controlling the motions of the simulator as well as the graphical display of the system. Conclusion and future works of this project are given in Section 5.

\section{Architecture}

This section describes the architecture and the major features of the virtual cycling simulator. Figure 2 illustrates the architecture of the simulator. Based on the motions of the bike, corresponding signals are transmitted to the PC controlling the system. The signals indicate the rotating angle of the front wheel and the speed of the rear wheel. The signal is transmitted through a serial port. The main control system decodes the received signal, and computes the necessary resistive torque required for the actuators. Meanwhile, the corresponding virtual environment is computed and rendered. Signal is transmitted to the projectors to display the scene.

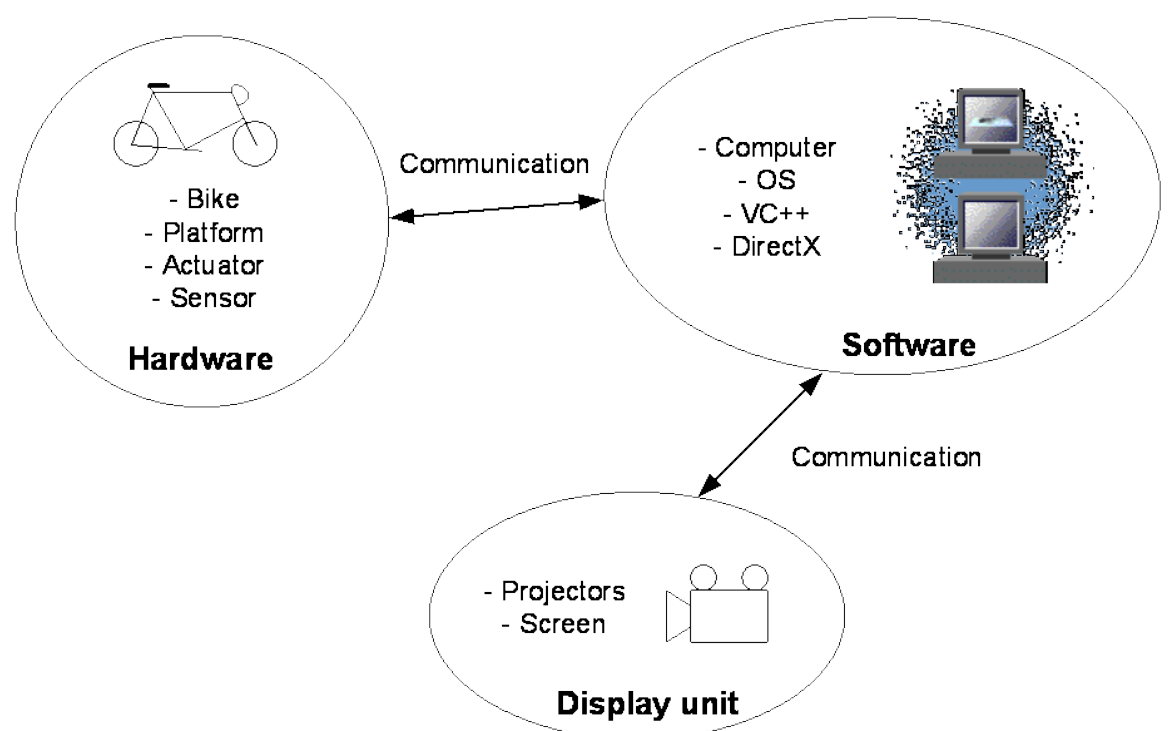

Fig. 2. The architecture of the virtual cycling simulator. The hardware of the simulator includes a bike platform, an actuation unit, a sensing unit and a display unit. The software communicates between the hardware and controls the necessary motions of the hardware. 
The simulator has the following major features:

1. Information including the speed of the bike and the distance traveled is displayed onto the screen. It allows evaluating the performance of the riders.

2. Capable of simulating the resistance due to different road frictions.

3. Capable of simulating the effect of riding up and down hill.

4. It allows small magnitude of tilting effect.

5. It provides sound effect during cycling.

6. The virtual scene can be displayed with stereoscopic effect.

\section{Hardware}

In this section, the hardware setup of the virtual cycling simulator is described. The hardware of the simulator consists of four major units including a bike platform, an actuation unit, a sensing unit and a display unit.

\subsection{Bike platform}

The bike platform consists of a rear wheel mounting stand, revising stand for the front wheel, a platform and six springs installed at the bottom of the platform. The rear wheel mounting stand is used to support and hold the bike. The revising stand supports and allows rotation of the front wheel of the bike. The springs installed at the bottom of the platform allows a small magnitude of tilting effect during cycling (Figure 3).

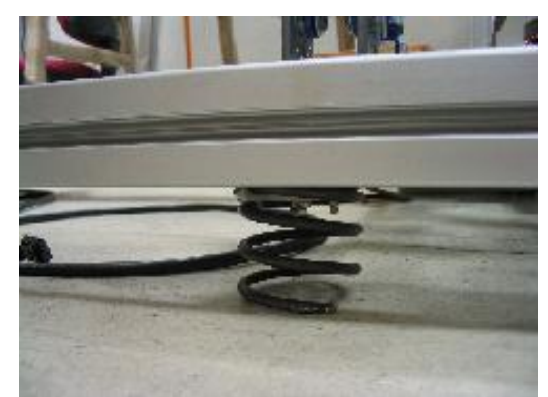

Fig. 3. Springs installed at the bottom of the platform allow small magnitude of titling during cycling.

\subsection{Actuation unit}

The actuation unit consists of a damper and a servomotor (Figure 4). The damper installed under the rear wheel is used to provide passive external resistance to the rear wheel. It is used to simulate the effect of various road frictions and the air resistance during cycling. The servomotor installed at the rear wheel generates different decelerating or active accelerating torque to the rear wheel based on the dynamic 
changes of the virtual environment. It is used to simulate the acceleration of the bike going downhill due to gravity and the deceleration effect when going uphill. Figure 5 shows a block diagram of the actuation unit. A closed-loop feedback control system is used to control the speed of the bike. Denote $\dot{\theta}_{\text {in }}$ and $\dot{\theta}_{\text {out }}$ as the input and output angular speed respectively. Given the initial speed of the bike $\dot{\theta}_{i n}$ and the inclination of the road, the required decelerating/ accelerating torque $\tau$ is computed and generated by the motor. This torque $\tau$ and the torque $\tau_{\text {user }}$ generated by the rider drive the rear wheel of which angular speed $\dot{\theta}_{\text {out }}$ is feedback to the control system.

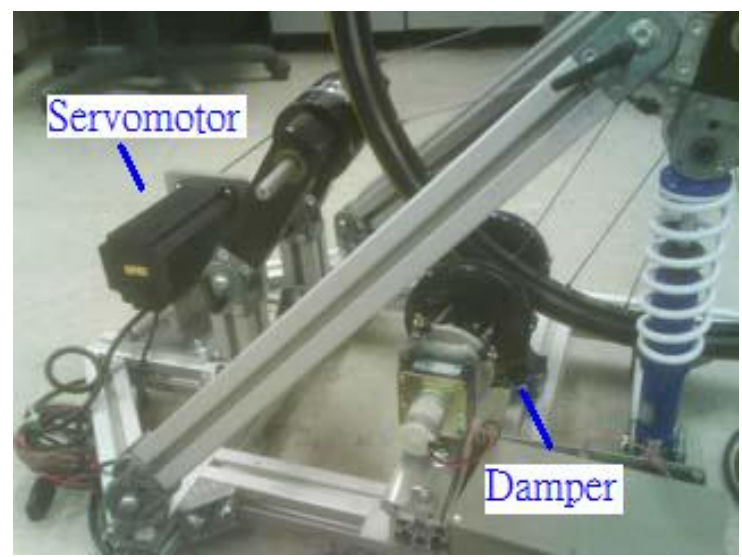

Fig. 4. The actuation unit consists of a servomotor generating active accelerating torque for the rear wheel.

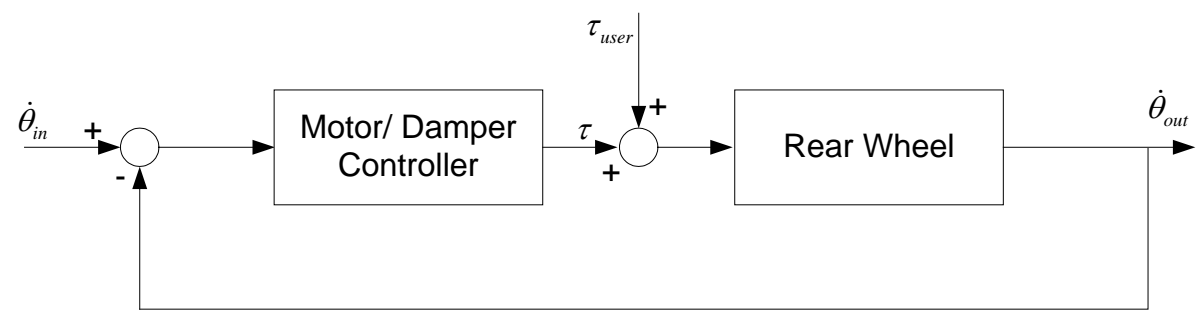

Fig. 5. Block diagram of the actuation unit.

\subsection{Sensing unit}

Two different data are required to be measured on the bike. These data include the angle of rotation and the speed of the bike. Two encoders are installed in the motors of the front and rear wheels of the bike. The encoder installed in the front wheel is used to measure the turning angle of the front wheel. The speed of the bike is measured by the encoder installed in the servomotor at the rear wheel. The measured signals are transmitted through a serial to the PC. 


\subsection{Display unit}

To display a stereoscopic image of the virtual environment, two projectors (Figure 6) are adopted to produce the stereoscopic effect of the virtual scene. One projector is responsible for projecting the image of the left eye and the other projector is responsible for projecting the image of the right eye. The rider is required to wear a $3 \mathrm{D}$ glass to perceive the stereoscopic effect.

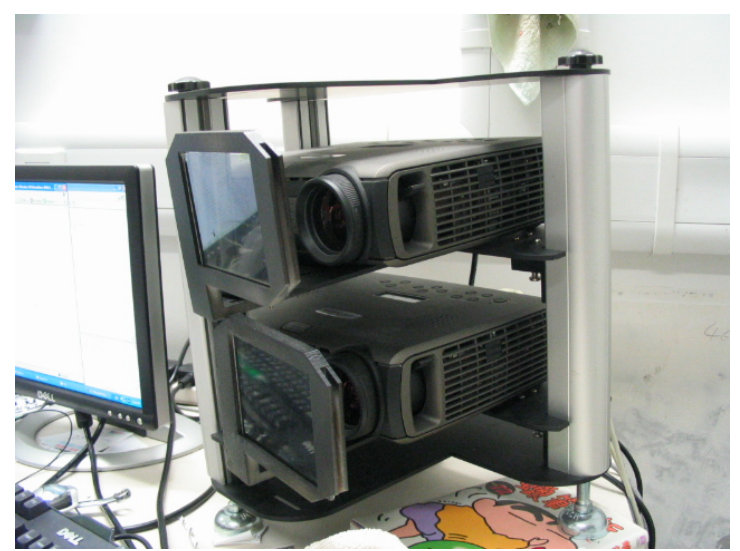

Fig. 6. Two projectors are used to produce the stereoscopic effect.

\section{Software}

The main control system is developed using Microsoft Visual C++. It serves two major functions in the system: communication and computation. For the communication, it receives signals from the sensing unit and transmits the signals to the actuation unit to control the motions of the motor. At the same time, signal is transmitted to the display unit to display the virtual environment. The main control system decodes the received signal and computes the necessary torque for the motor. The dynamic changes in the environment and the bike are considered in the computation to control the actuation unit. Meanwhile, the corresponding virtual environment is computed and rendered. Section 4.1 discusses the bike dynamics and the virtual scene used in our system is discussed in Section 4.2.

\subsection{Bike dynamics}

The dynamic model of the bike is the key component in the software system of an interactive cycling simulator. It solves the motion equations that account for the geometrical and physical characteristics of the simulator. Studies of the bike dynamics can be found in [12] and [13]. In He et al. [13], a detail analysis of the bike dynamics is described. In their study, forces acting on the front and rear wheel of the bike are formulated. The tilting torque and the frictional force of different road 
profiles are also considered. In our model, the bike is divided into the front and rear parts represented by two different vectors. A vector is used to represents the direction of the motion and the other vector represents the magnitude of the current velocity of the bike. Figure 7 illustrates the notations and representations of the bike vector model. The angle of rotation of the bike $\theta$ is determined by the angles between the vectors representing the front part $\mathbf{v}_{1}$ and the rear part $\mathbf{v}_{2}$. The main control system computes the magnitude of the vector of the rear part $\left\|\mathbf{v}_{2}\right\|$ according to the dynamic changes in the frictional force and the inclination of the road. The computed data is used to control the velocity of the bike and the required torque generated by the servomotor.

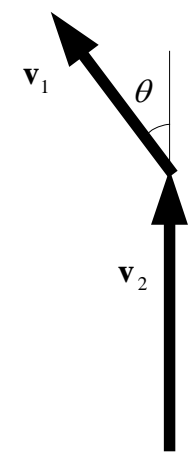

Fig. 7. Two vectors representing the front part $\mathbf{v}_{1}$ and the rear part $\mathbf{v}_{2}$ of the bike are adopted. $\theta$ is used to represent the angle of turn and $\left\|\mathbf{v}_{2}\right\|$ is used to represent the current velocity of the bike.

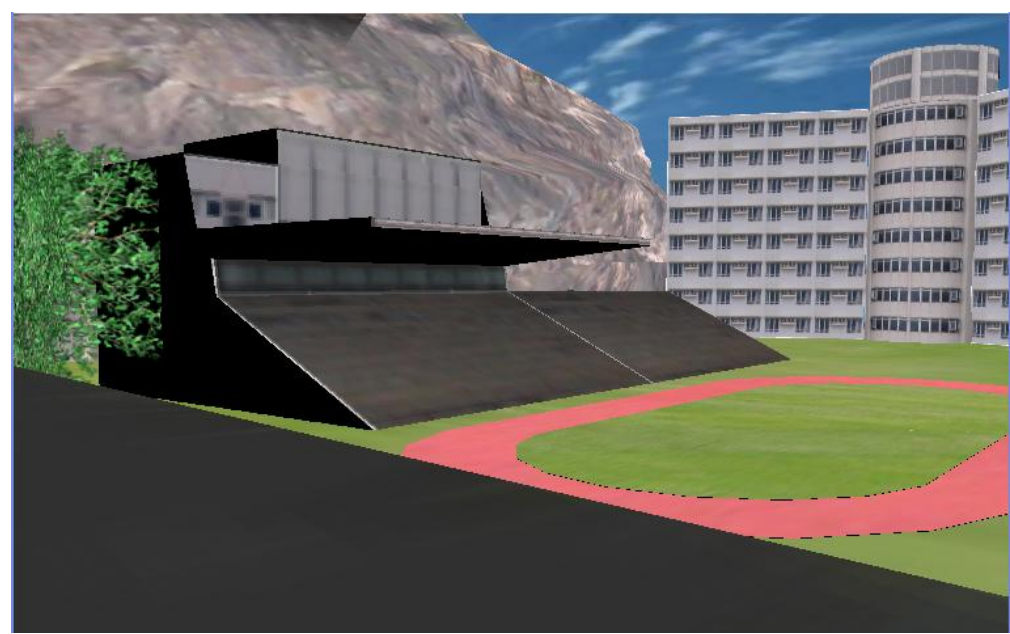

Fig. 8. The virtual environment of the university gymnasium in the Chinese University of Hong Kong. 


\subsection{The virtual scene}

In the proposed system, DirectX 9.0 is adopted as the graphics engine for displaying the virtual scene. A 3D model of the virtual scene is created by using existing CAD software. The virtual environment of the university gymnasium in the Chinese University of Hong Kong is constructed (Figure 8). The virtual environment including a slope is designed for the cyclers to experience the feeling of riding up/ down hill (Figure 9).

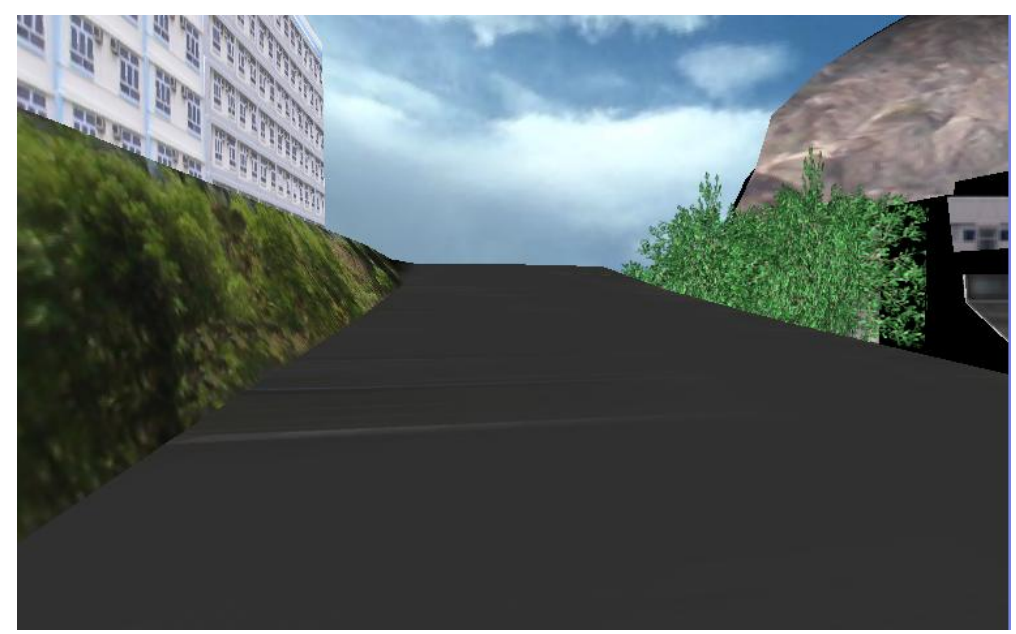

Fig. 9. A slope is designed for the cyclers to experience riding up/ down hill.

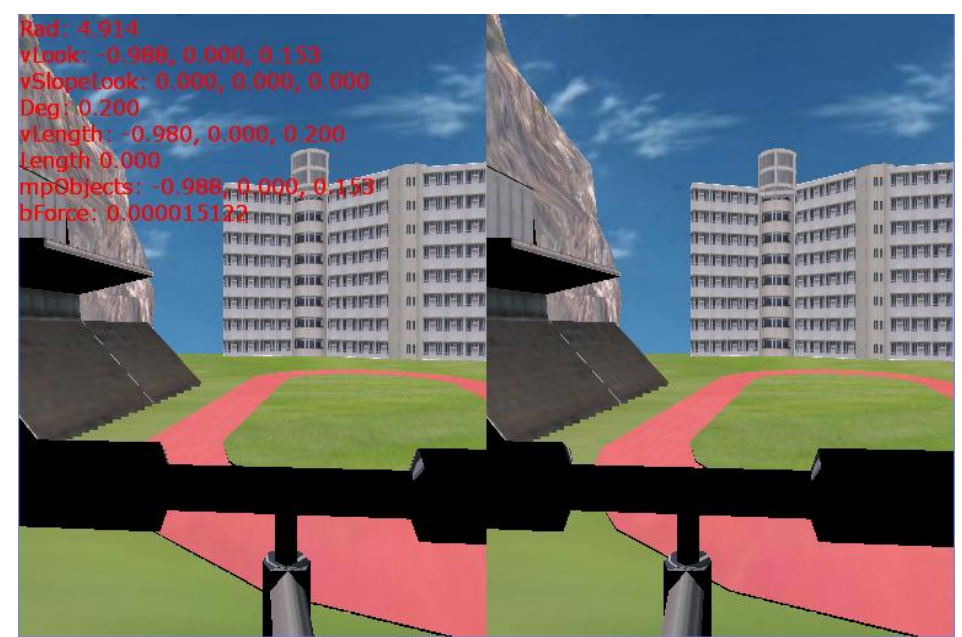

Fig. 10. Two scenes are computed and rendered at the same time to produce the stereoscopic effect.

The simulator computes the dynamic change of the virtual environment and renders two scenes at the same time. One scene displays the environment for the left 
eye and another scene displays the environment for the right eye, which are projected with two separate projectors. Figure 10 illustrates the two scenes images of the left and right eyes. Meanwhile, the instantaneous speed of the bike and the distance traveled is computed and displayed on the screen.

\section{Conclusion and Future Works}

A virtual cycling simulator which is suitable for exercise and entertainment has been developed. The simulator allows cyclers to travel in the virtual environment of the university gymnasium in the Chinese University of Hong Kong. The simulator is capable of simulating the dynamic changes of the virtual environment including the resistance of different land profile and the decelerating/ accelerating effect of the bike when going up/ down hill. To increase the realism of the virtual scene, stereoscopic effect is also provided. The speed of the bike and the distance traveled are computed and displayed on the screen for evaluating the performance of the cyclers. In this system, a single PC with dual display output is required for controlling the actuation unit and for the graphics display.

To increase the realism of the cycling experience, it is expected that more outdoor cycling elements such as wind and sunlight effects can be included in the system. To further enhance the system for exercise purpose, the system can be extended into a multi-players system. In addition, various playing modes such as training and competition modes can be introduced. We may also develop virtual environments of some famous places in Hong Kong such as Mong Kok and Tsim Sha Tsui or other parts of the world to arouse more interest in using the system.

\section{References}

1. Hillman, M.: Cycling towards health and safety. London: BMA (1994)

2. Carnall, D.: Cycling and health promotion. BMJ Vol. 320 (2000) 888

3. VRML Virtual Reality Modeling Language, http://www.w3.org/MarkUp/VRML/

4. VRML game, http://www.ceres.dti.ne.jp/ kekenken/main/3d/game/game.htm

5. Interactive realtime 3D software, http://www.bitmanagement.com/

6. CompuTrainer MultiRider systems, http://www.computrainer.com/multirider_fun.htm

7. CompuTrainer, http://www.racermateinc.com/

8. Carraro, GU..: The Peloton Bicycling simulator. Proceedings of the $3^{\text {rd }}$ symposium on Virtual reality modeling language. Monterey, California, United States (1998) 63-70

9. Carraro, G.U., Edmark, J.T. and Ensor, J.R.: Techniques for Handling Video in Virtual Environments. Proceedings of the 25th annual conference on Computer graphics and interactive techniques. Orlando, Florida, Addison Wesley (1998) 353-360

10. Kwon, D.S., Yang, G.H., et al.: KAIST Interactive Bicycle Simulator. Proc. of the IEEE International Conference on Robotics and Automation. Korea Seoul Vol. 3 (2001) 23132318

11. Westerink, J.H.D.M., Jager, M. de, Herk, J. van, Kort, Y.A.W. de, IJsselsteijn, W.A..: The influence of virtual coaching on social presence and motivation in home fitnessing. Psychology and Health 19, (2004) 181-182 
12. Hand, R.H.: Comparisons and Stability Analysis of Linearized Equations of Motion for a Basic Bicycle Model. M.S. thesis, Cornell University, Cornell, NY (1988)

13. He, Q., Fan, X. and Ma, D.: Full Bicycle Dynamic Model for Interactive Bicycle Simulator. Journal of Computing and Information Science in Engineering Vol. 5 Issue 4, (2005) 373380 